\title{
Prototipo electrónico para el calentamiento a baja frecuencia de transformadores eléctricos monofásicos
}

\author{
Diego F. García, Hernando Vásquez* y Andrés M. Holguín \\ Facultad de Ingeniería, Escuela de Ingeniería Eléctrica y Electrónica, Universidad del Valle, Calle 13 \#100-00, Cali, \\ Colombia (correo-e: diego.garcia@correounivalle.edu.co; hernando.vasquez@correounivalle.edu.co; \\ andres.holguin@correounivalle.edu.co) \\ * Autor a quien debe ser dirigida la correspondencia.
}

Recibido Ene. 22, 2020; Aceptado Mar. 24, 2020; Versión final May. 21, 2020, Publicado Oct. 2020

\begin{abstract}
Resumen
En este trabajo se presenta el desarrollo de un prototipo de sistema electrónico para coadyuvar en el secado de transformadores eléctricos monofásicos mediante la inyección de corrientes sinusoidales de baja frecuencia. El prototipo desarrollado está conformado principalmente por un cicloconvertidor controlado a través de una matriz de puertas lógicas programable en campo. El prototipo incluye estructuras de control y regulación de las variables eléctricas y térmicas asociadas con el calentamiento del transformador bajo secado. Para el desarrollo del prototipo se partió de un diseño básico, el cual se modelizó en MATLAB, para, a partir de los resultados de las simulaciones, realizar los ajustes al diseño, así como evaluar las estrategias de control y de estimación de la temperatura del transformador bajo calentamiento. Finalmente, el funcionamiento del sistema construido, incluyendo el control de la temperatura de calentamiento del transformador bajo secado, fueron evaluados experimentalmente mediante pruebas de laboratorio.
\end{abstract}

Palabras clave: cicloconvertidor; FPGA; control de temperatura, calentamiento a baja frecuencia; transformadores

\section{Electronic prototype for low-frequency heating of single-phase electrical transformers}

\begin{abstract}
This work presents the development of an electronic system prototype to assist in the drying of single-phase electrical transformers by injecting sinusoidal low-frequency currents. The main component of the prototype developed here is a cyclo-converter, which is controlled through a field programmable gate array. The prototype includes control and regulation structures for the electrical and thermal variables that are associated with heating of the transformer under drying. A basic design modeled in MATLAB was used to develop the proposed system. Based on the simulation results, the design was adjusted and the control and estimation strategies of the transformer's temperature under heating were evaluated. The operation of the system, including the control of the heating temperature of the transformer under drying, was experimentally assessed by laboratory tests.
\end{abstract}

Keywords: cycloconverter; FPGA; temperature-control, low frequency heating; transformers 


\section{INTRODUCCIÓN}

La humedad y la temperatura son dos de los factores que inciden sobre la degradación de los aislamientos celulósicos y por ende en la expectativa de vida de los transformadores eléctricos (Cerón et al., 2015; Martínez et al., 2019); la temperatura está relacionada directamente con la operación del transformador, en tanto que la humedad es considerada un contaminante. Para contribuir a reducir el contenido de humedad en los aislamientos celulósicos en transformadores de mediana y alta potencia, es necesario someter al transformador a un secado artificial, en el cual, la tasa de extracción de la humedad aumenta con el incremento de temperatura en los aislamientos.

Durante el secado del transformador, una de las formas de mejorar el aporte calórico a sus aislamientos celulósicos, incrementando su temperatura, es hacer circular corrientes por sus devanados (García et al., 2012). El calor generado por las pérdidas en los devanados del transformador es conducido a los aislamientos celulósicos en contacto con los conductores y por convección o radiación, dependiendo del método de secado, a los otros aislamientos celulósicos, favoreciendo así el secado de estos (García et al., 2011). Adicionalmente, con esta técnica el flujo de calor se produce en la misma dirección de la difusión de humedad. Para lograr la circulación de corrientes por los devanados del transformador, estando este fuera de servicio se suele aplicar tensión alterna en uno de sus devanados, mientras al menos otro de los devanados está en cortocircuito. Con ello circula corriente por el devanado alimentado y se inducen corrientes en los devanados en cortocircuito. Bajo estas condiciones la fuente empleada para el calentamiento ve al transformador como una impedancia predominantemente inductiva, definida por su impedancia de cortocircuito, $Z_{c c}=R_{c c}+j X_{c c}$, donde la componente de mayor peso es la reactancia de cortocircuito, $X_{c c}=2 \pi f L_{c}$, la cual es función de la frecuencia de la señal de alimentación. La impedancia de cortocircuito define la tensión y potencia requeridas de la fuente para producir el calentamiento. En transformadores de potencia, la fuente requerida para hacer circular corrientes, cuya magnitud garantice un calentamiento adecuado de los aislamientos sólidos, puede llegar a ser de considerable tamaño, con lo cual pierde practicidad este método de calentamiento.

Una forma de reducir el tamaño de la fuente empleada para el calentamiento de transformadores de potencia, durante el secado en campo, es reduciendo la frecuencia de la señal de tensión aplicada, en lo que se conoce como calentamiento de baja frecuencia o LFH por sus siglas en inglés (Low Frequency Heating) (Koestinger et al., 2004; Vuolle y Rindlisbacher, 2005; Koestinger y Bruarøy, 2006; Figueroa et al., 2009; Dahlund et al., 2010), con lo cual se logra reducir la componente inductiva de la impedancia de cortocircuito, permitiendo que el sistema alimente una carga fundamentalmente resistiva. El LFH se constituye por tanto en un método coadyuvante en los diferentes tipos de secado convencionales que se utilizan en los transformadores.

Una opción para obtener las señales de baja frecuencia es el empleo de controladores de velocidad variable o VSD por sus siglas en inglés (variable speed drivers), como los usados en el control de velocidad de motores (Skinner, 2013); sin embargo, al ser una aplicación distinta para la cual fueron construidos, este tipo de equipos convertidores AC-DC-AC, basados en el principio de modulación de ancho de pulso (PWM), por la distorsión de las señales de salida, no facilitan el control de temperatura durante el calentamiento del transformador, además de que a las frecuencias en que se desea aplicar la técnica LFH (inferiores a $5 \mathrm{~Hz}$ ), este tipo de variadores no pueden entregar su tensión nominal, con lo cual es necesario sobredimensionar el equipo. Un trabajo más reciente da cuenta del diseño de un equipo para calentamiento a baja frecuencia de transformadores bajo secado basado en convertidores AC-DC-AC, de puentes de rectificadores no controlados (diodos), que hace un seguimiento de la corriente de baja frecuencia de la señal de referencia mediante teoría de control de pasividad (Zhu et al., 2015).

El sistema LFH propuesto para la generación de señales de baja frecuencia para el calentamiento del transformador bajo secado, se basa en un convertidor AC-AC, que es un puente trifásico dual de tiristores o cicloconvertidor (Mohan et al., 2009) y cuyo sistema de control permite regular la temperatura de los devanados durante el proceso de calentamiento del transformador bajo secado. En este caso, el control de temperatura se basa en estimar el cambio de la resistencia de los devanados con la temperatura (Naimi et al., 2013).

\section{METODOLOGÍA}

La metodología desarrollada se presenta en cuatro subsecciones: primero se describe el sistema propuesto, luego explica el convertidor de potencia; posteriormente se presenta el modelo de simulación empleado para el ajuste del diseño propuesto y por último se relata la implementación del control del sistema LFH.

\section{Descripción del sistema LFH propuesto}

El prototipo de sistema LFH propuesto tiene una salida monofásica con una potencia de $1 \mathrm{kVA}$, con tensión de salida de hasta $100 \mathrm{~V}$, capaz de entregar una corriente sinusoidal de $10 \mathrm{~A}$, con frecuencias que van desde 
0,1 a $5 \mathrm{~Hz}$. Este sistema LFH consta de dos etapas principales: una de potencia, con la que se logra la inyección de corrientes de baja frecuencia al transformador a secar y otra de control, con la que se maneja todo el sistema y se protege el accionar del mismo.

Para la etapa de potencia se definió el uso de un cicloconvertidor monofásico, el cual es un convertidor ACAC, que permite la producción de señales de tensión y corriente, de muy baja frecuencia, con la posibilidad de controlar su amplitud. Para la etapa de control se utilizó una matriz de puertas lógicas programable en campo o FPGA por sus siglas en inglés (Field-Programmable Gate Array). En la Figura 1 se muestra el esquema general del sistema LFH propuesto, allí además del cicloconvertidor y la FPGA se observan otros elementos indispensables para el funcionamiento de este, como son el bloque "Sensores y filtrado" con el que se adecuan, para su registro, las señales de tensión y corriente que se entregan al transformador bajo secado. Estas señales de tensión y corriente se digitalizan en el bloque "Conversión análogo a digital", para ser ingresadas a la FPGA; también se aprecia un bloque "Detector de cruce por cero", cuya señal emplea la FPGA para generar los pulsos de disparo de los tiristores del cicloconvertidor. El sistema, a través de la FPGA, tiene comunicación con un PC, desde el cual el usuario puede operar el mismo.

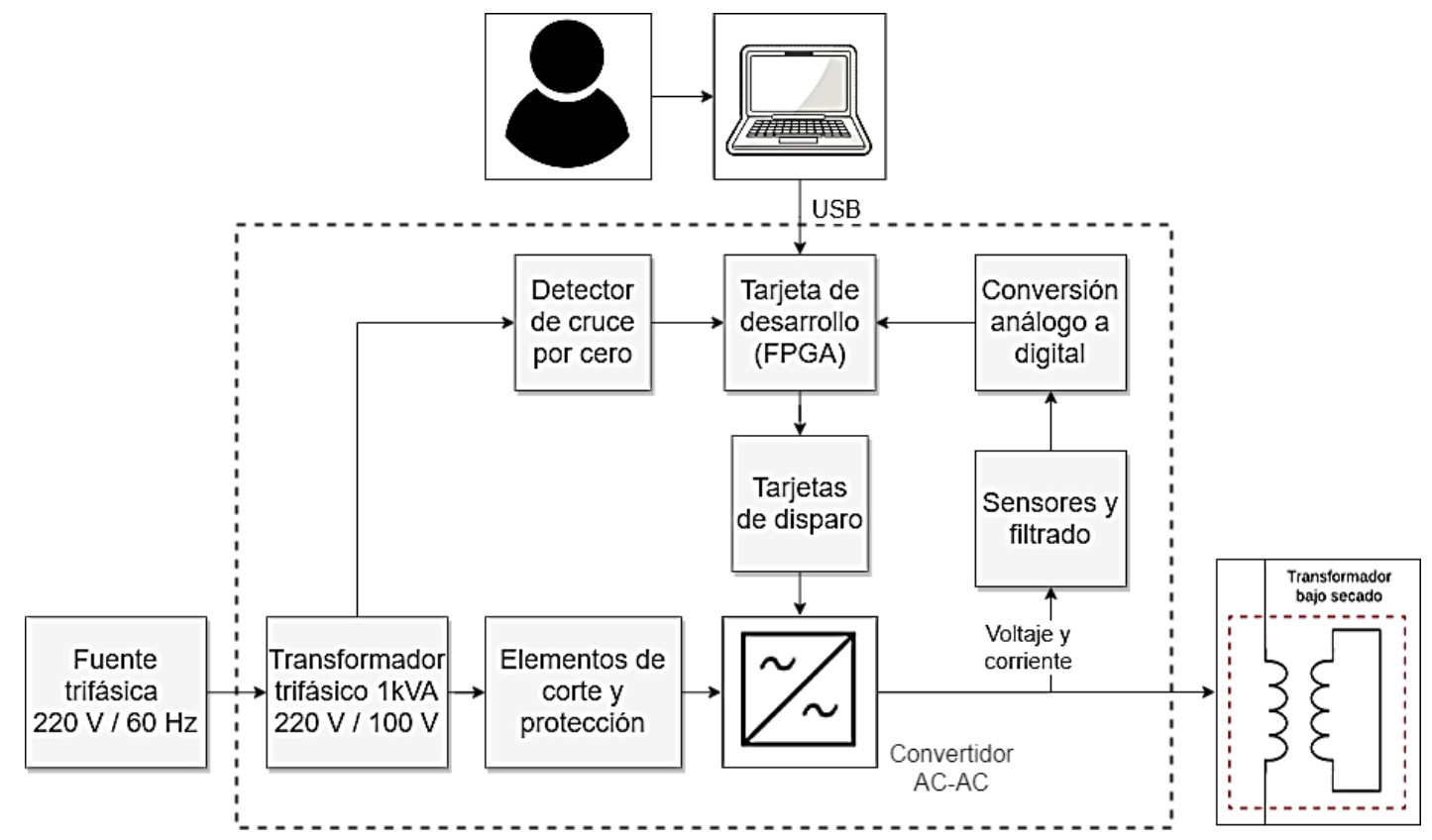

Fig 1: Esquema general del sistema LFH.

\section{Cicloconvertidor}

El requerimiento básico del sistema LFH es entregarle a la carga corrientes sinusoidales de muy baja frecuencia, para lo cual se decidió utilizar el cicloconvertidor, cuyo modelo se muestra en la Figura 2. El cicloconvertidor consta de dos puentes trifásicos de tiristores, cada uno de ellos maneja la mitad de un ciclo de la señal de corriente sinusoidal que se le aplica al transformador bajo secado, lo cual categoriza a éste como un cicloconvertidor sin corriente circulante.

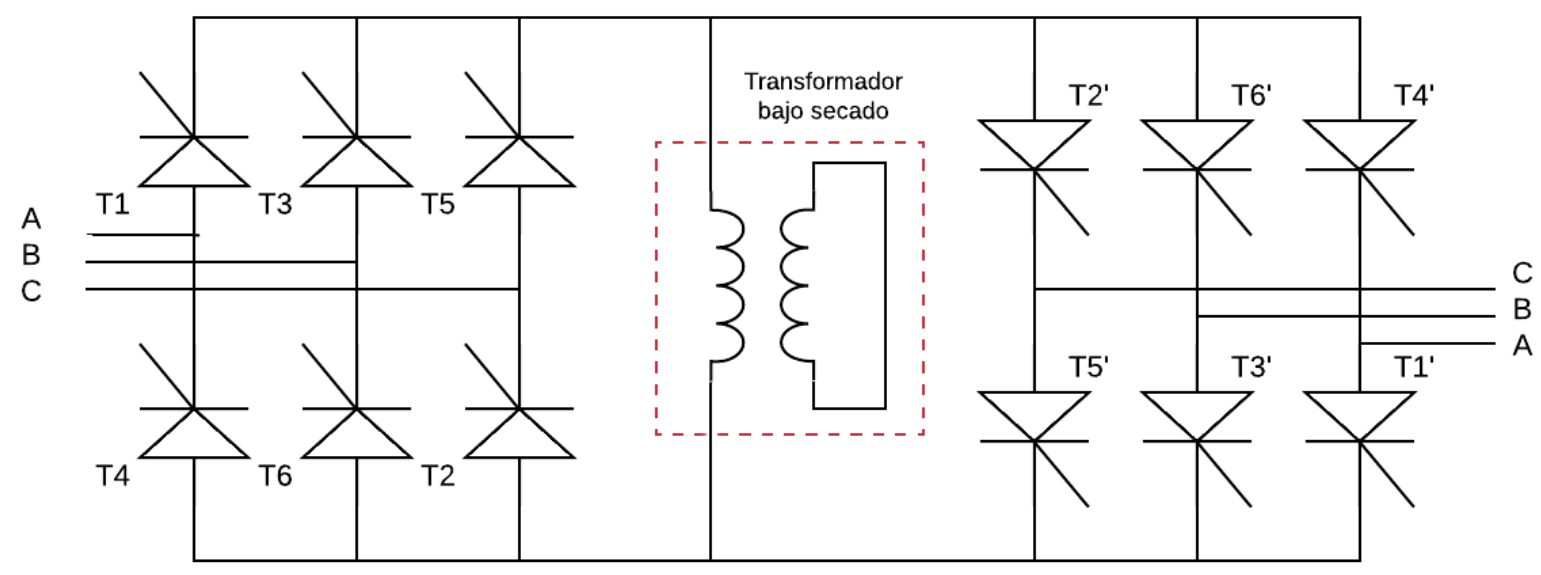

Fig 2: Esquema básico del cicloconvertidor utilizado. 
El cicloconvertidor es alimentado desde un sistema trifásico equilibrado, por lo cual, la ecuación que caracteriza el voltaje de salida, $\mathrm{V}_{\mathrm{cc}}$, de cada uno de los puentes trifásicos de tiristores es:

$$
\mathrm{V}_{\mathrm{cc}}=\frac{3 \sqrt{3} \mathrm{~V}_{\max }}{\pi} \cos (\alpha)
$$

Siendo $V_{\max }$ el voltaje máximo de la señal de voltaje $A C$ aplicada y a el ángulo de disparo, con el cual se puede modificar el voltaje que se le aplica a la carga. Cada puente rectificador puede manejar ángulos de disparo $\alpha$, entre $0^{\circ}$ y $180^{\circ}$. El valor $\alpha$ de un puente, es el ángulo complementario del valor $\alpha$ del otro puente.

\section{Modelo de simulación del sistema LFH}

Para ajustar y validar el diseño propuesto, se implementó un modelo del sistema LFH en Matlab. A través de este modelo se determinaron las características requeridas de los tiristores del cicloconvertidor, del transformador de alimentación, de los elementos de protección y corte, de los sensores y los filtros. El modelo de simulación permitió además evaluar las estrategias de control de la corriente y la estimación de la temperatura de los devanados del transformador bajo secado. En la Figura 3 se muestra el esquema del modelo implementado.

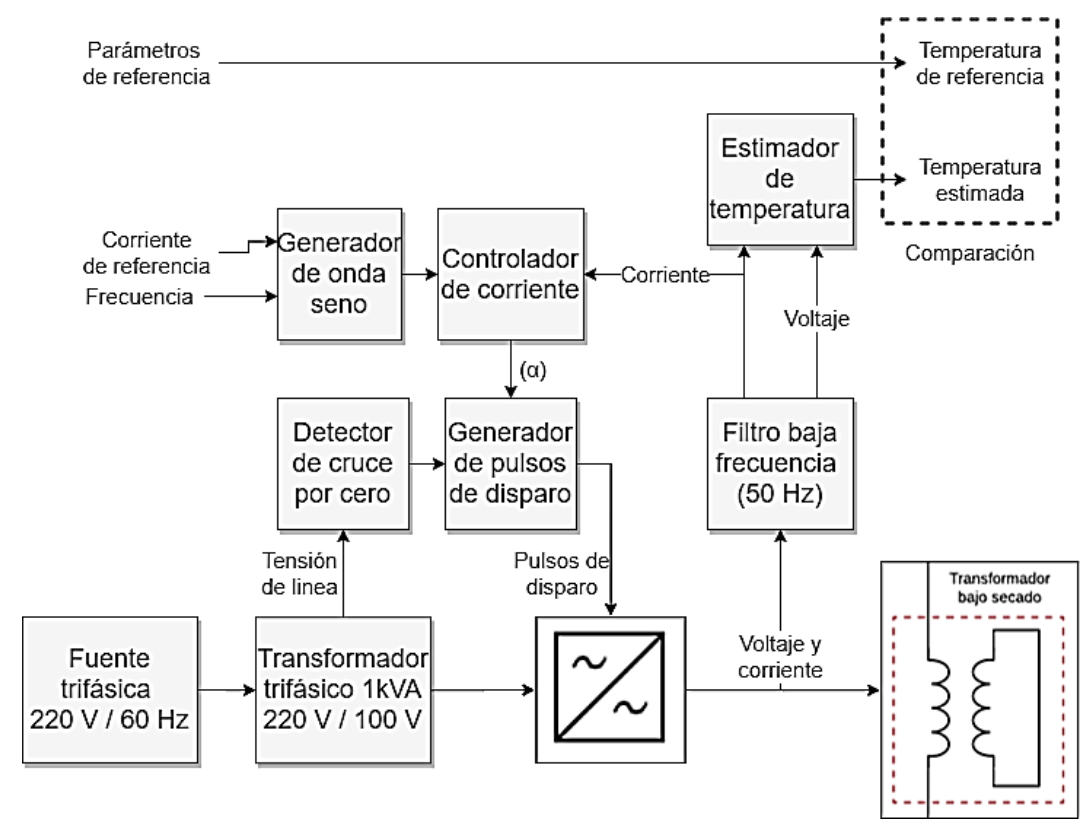

Fig 3: Diagrama esquemático del modelo de Matlab implementado.

El control de temperatura no se incluyó en el modelo de Matlab, debido a que la dinámica térmica de un transformador tiene unas constantes de tiempo del orden de minutos e incluso horas y, por tanto, no es posible la simulación de esta dinámica, en simultáneo con la del control. En el modelo de simulación, el efecto del comportamiento térmico del transformador bajo secado es tenido en cuenta por medio de la corriente de referencia qué, a través del "Generador de onda seno" llega al "Controlador de corriente". Adicionalmente, el transformador bajo secado se modeló como una impedancia constante cuyo valor depende de la temperatura que se pretende estimar.

En el diagrama de la Figura 3, el convertidor se modeló como dos rectificadores controlados conectados en antiparalelo, cuyas señales de encendido provienen del generador de pulsos de disparo, el cual emplea la señal del ángulo de disparo ( $\alpha$ ) proveniente del "Controlador de corriente", y la señal de sincronización del "Detector de cruce por cero". En la Figura 4 se muestran los pulsos de disparo para uno de los puentes de tiristores requeridos para obtener señales de salida del sistema LFH, cada gráfico horizontal corresponde a la señal de activación de un tiristor; se observa que hay un doble pulso para cada tiristor, esto se hace para asegurar la activación de la pareja de tiristores correspondiente.

La inyección de corriente al transformador bajo secado debe controlarse para que la temperatura de los devanados no supere la temperatura máxima permitida, según las especificaciones del transformador y por ello se incluye el "Controlador de corriente", el cual toma una referencia de corriente relacionada con la temperatura de los devanados del transformador y la frecuencia de referencia, proveniente del "Generador 
de onda seno". En la parte superior de la Figura 5 se muestran las señales de corriente de referencia (línea punteada) y salida (línea sólida) y en la parte inferior se presentan la tensión de salida y el ángulo de disparo (línea punteada), obtenidas del modelo de simulación para uno de los casos estudiados.
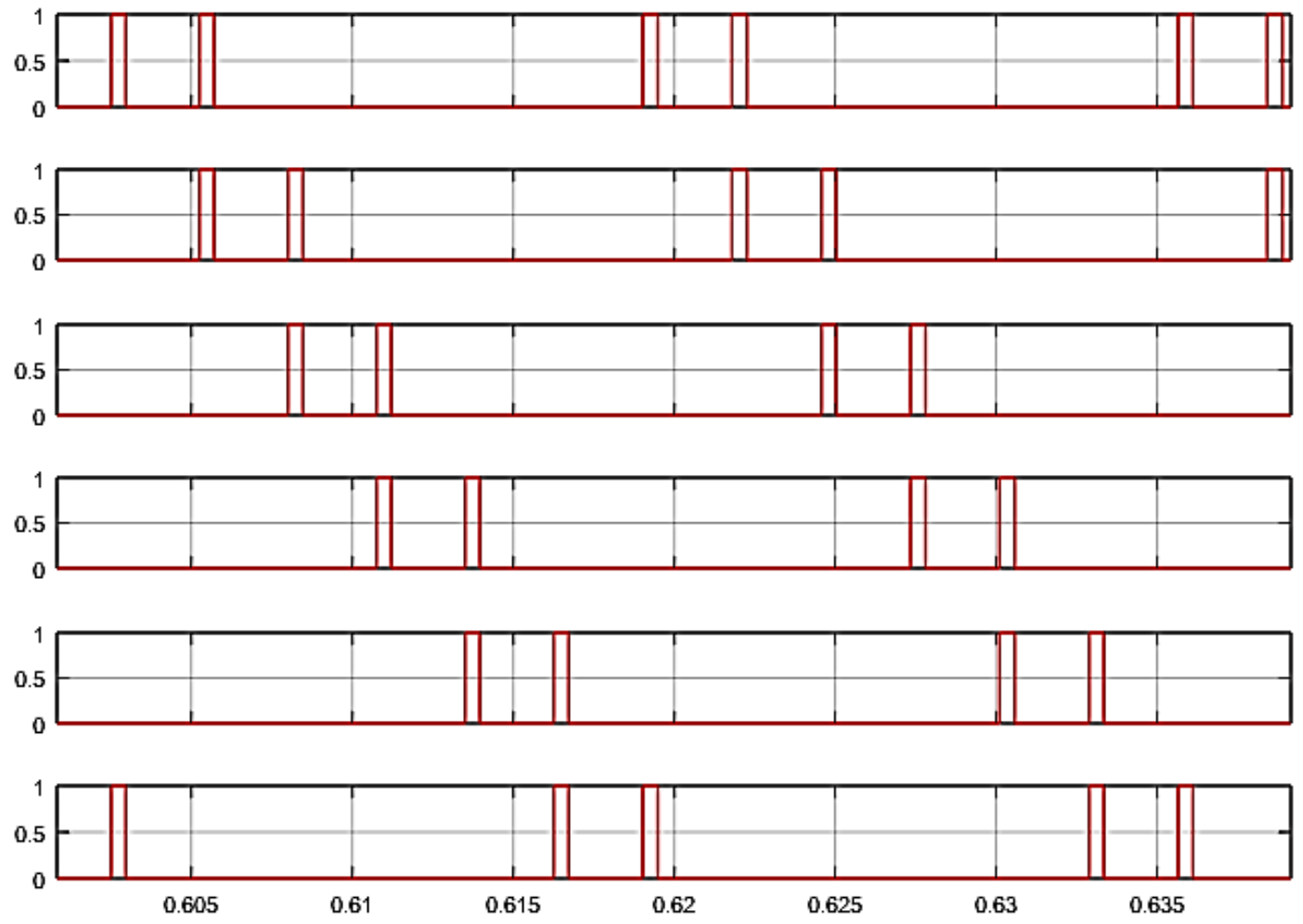

Fig 4: Pulsos de disparo requeridos en uno de los puentes de tiristores.
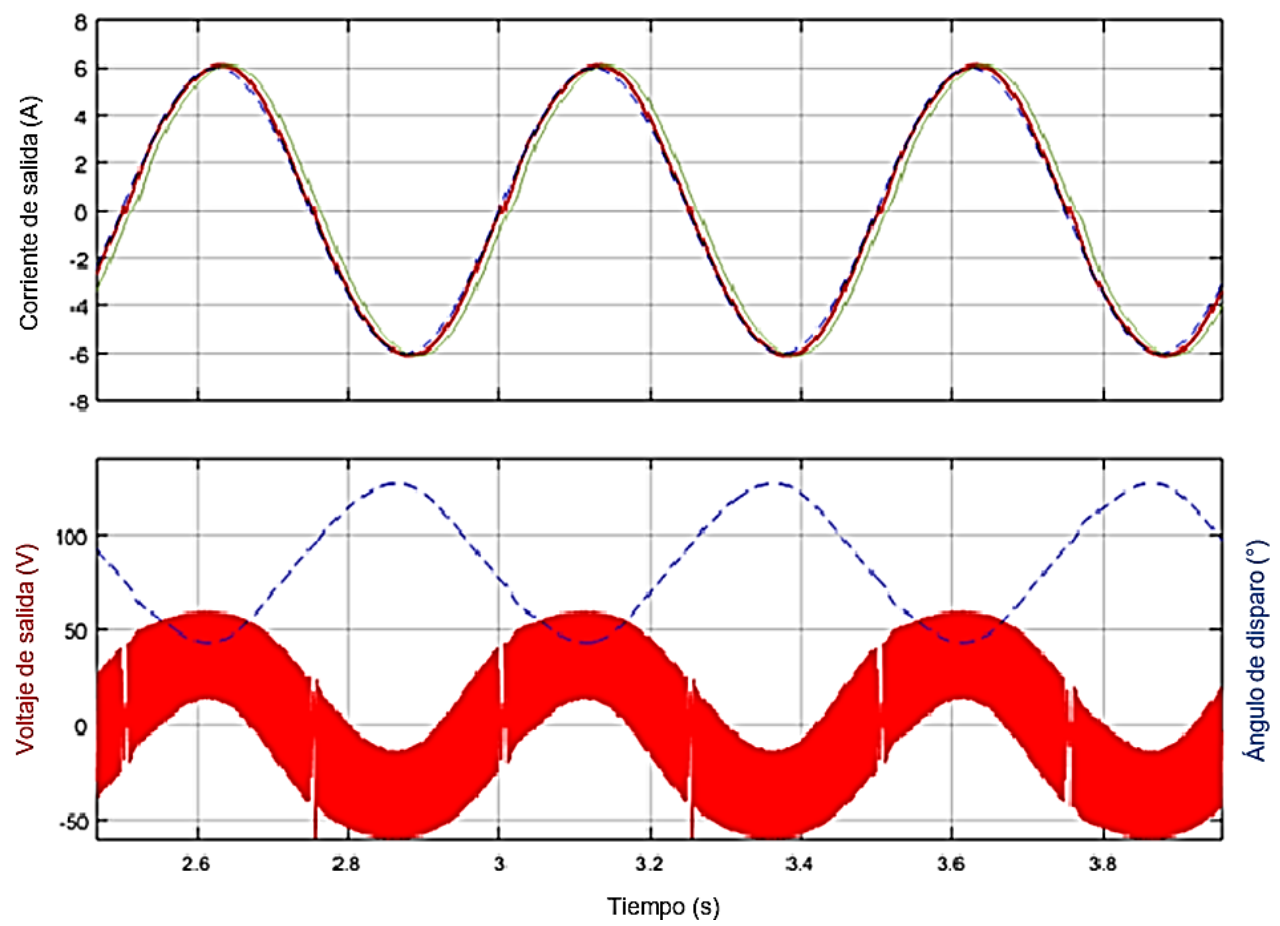

Fig 5: Corrientes de referencia y salida, tensión de salida y ángulo de disparo para un caso de estudio.

En el bloque "Estimador de temperatura", a partir de las señales de tensión y corriente de salida del sistema LFH, se estima de forma indirecta la temperatura de los devanados del transformador bajo secado, al calcular el cambio de su resistencia (Naimi, Farhangi y Ghaemi, 2003; IEEE, 2016), para ello se emplea la ecuación (2). 


$$
T_{s}=\frac{R_{s}}{R_{m}}\left(T_{m}+T_{k}\right)-T_{k}
$$

Siendo $T_{s}$ la temperatura estimada; $R_{s}$ es la resistencia del devanado, determinada a partir de las señales de tensión y corriente medidas; $\mathrm{Rm}_{\mathrm{m}}$ es la resistencia DC del transformador, medida en pruebas previas; $\mathrm{T}_{\mathrm{m}}$ es la temperatura a la que se midió la resistencia de DC de los devanados y $T_{k}$ es la temperatura característica, que depende del material de los devanados $\left(234,5^{\circ} \mathrm{C}\right.$ para cobre y $225^{\circ} \mathrm{C}$ para aluminio). Las señales de entrada de tensión y corriente que llegan al "Estimador de temperatura" requieren ser filtradas para que el cálculo de $\mathrm{R}_{\mathrm{s}} \mathrm{y}$, por ende, la estimación de temperatura sea precisa. En la Tabla 1 se presentan algunos resultados de estimación de la temperatura obtenidos a través del modelo de Matlab.

Tabla 1: Temperaturas estimadas a través del modelo de Matlab.

\begin{tabular}{|c|c|c|c|c|c|c|}
\hline \multicolumn{2}{|c|}{$\begin{array}{l}\text { Valores de salida } \\
\text { del sistema } L F H\end{array}$} & \multicolumn{2}{|c|}{$\begin{array}{l}\text { Valores asignados al } \\
\text { modelo del transformador }\end{array}$} & \multicolumn{2}{c|}{$\begin{array}{l}\text { Valores estimados en el } \\
\text { modelo de simulación }\end{array}$} & $\begin{array}{l}\text { Error relativo } \\
\text { entre las } \\
\text { temperaturas }(\%)\end{array}$ \\
\hline$F(H z)$ & $I$ pico $(A)$ & $T_{s}\left({ }^{\circ} \mathrm{C}\right)$ & $R_{s}\left({ }^{\circ} \mathrm{C}\right)$ & $R_{s}\left({ }^{\circ} \mathrm{C}\right)$ & $T_{s}\left({ }^{\circ} \mathrm{C}\right)$ & $-0,08$ \\
\hline 0,5 & 6 & 25 & 6,51 & 6,509 & 24,98 & $-0,68$ \\
\hline 1,0 & 6 & 25 & 6,51 & 6,506 & 24,83 & 1,20 \\
\hline 2,0 & 6 & 25 & 6,51 & 6,518 & 25,30 & $-9,28$ \\
\hline 3,0 & 6 & 25 & 6,51 & 6,450 & 22,68 & $-8,12$ \\
\hline 4,0 & 6 & 25 & 6,51 & 6,459 & 22,97 & 27,20 \\
\hline 5,0 & 6 & 25 & 6,51 & 6,679 & 31,80 & 58,20 \\
\hline 1,0 & 1 & 25 & 6,51 & 6,875 & 39,55 & 3,00 \\
\hline 1,0 & 2 & 25 & 6,51 & 6,529 & 25,75 & 2,00 \\
\hline 1,0 & 3 & 25 & 6,51 & 6,525 & 25,50 & 2,40 \\
\hline 1,0 & 4 & 25 & 6,51 & 6,525 & 25,60 & 0,08 \\
\hline 1,0 & 5 & 25 & 6,51 & 6,511 & 25,02 & 1,00 \\
\hline 1,0 & 6 & 25 & 6,51 & 6,516 & 25,25 & 1,04 \\
\hline 1,0 & 7 & 25 & 6,51 & 6,517 & 25,26 & 0,04 \\
\hline 1,0 & 8 & 25 & 6,51 & 6,510 & 25,01 & \\
\hline
\end{tabular}

En la Tabla 1 se observa que los mayores errores en la estimación de la temperatura de los devanados del transformador bajo secado se producen en dos situaciones: cuando la frecuencia de la señal corresponde a la máxima frecuencia $(5 \mathrm{~Hz})$ y cuando la corriente de salida es muy baja, momento en que el funcionamiento de los puentes de tiristores es discontinuo. En ambas situaciones, la señal de corriente de salida tiene una alta distorsión, lo que afecta la estimación de la temperatura porque se dificulta determinar con precisión el desfase entre las señales de tensión y de corriente.

Para mitigar esta situación se requiere en primera medida contar con un transformador de alimentación con derivaciones, para así aplicar un valor de tensión apropiado al cicloconvertidor, según los requerimientos del transformador bajo secado, de tal manera que la excursión del ángulo de disparo sea mayor y con ello se reduzca la distorsión de la señal de corriente. De otra parte, es recomendable usar el equipo LFH con frecuencias de salida cercanas al límite inferior, lo que además es beneficioso porque implica un menor requerimiento de potencia durante el proceso de secado.

\section{Control del sistema $L F H$}

Como se mencionó anteriormente, para el control del sistema LFH se usó la FPGA Terasic, modelo DE1SoC, la cual es un dispositivo programable que contiene bloques de lógica cuya interconexión y funcionalidad puede ser configurada a nivel de hardware mediante un lenguaje de descripción especializado. Con la FPGA se ejecutan todas las acciones de control del equipo, así como las acciones de decisión y protección de este. Dentro de las funciones implementadas en la FPGA se pueden enumerar: a) la definición de la frecuencia de trabajo, entre 0,1 y $5 \mathrm{~Hz}$; b) la generación de los pulsos de disparo para cada uno de los tiristores del cicloconvertidor; c) programación del comportamiento del cicloconvertidor, para que entregue en su salida una señal sinusoidal de corriente, a las frecuencias requeridas; d) programación del bucle interno de control de corriente, con el cual se puede garantizar la aplicación al transformador bajo secado, de una corriente sinusoidal con los valores máximo y de frecuencia especificados por el usuario; e) control indirecto de la temperatura de calentamiento de los devanados del transformador; f) conexión con el computador, lo que permite definir externamente los valores de frecuencia y voltaje que se le aplicarán al transformador bajo secado; g) procesamiento del voltaje y la corriente que se le aplica al transformador, lo cual permite calcular la temperatura a la cual están siendo sometidos los devanados del transformador bajo secado. En la Figura 6 se muestra el esquema con los bloques funcionales implementados en la FPGA. 


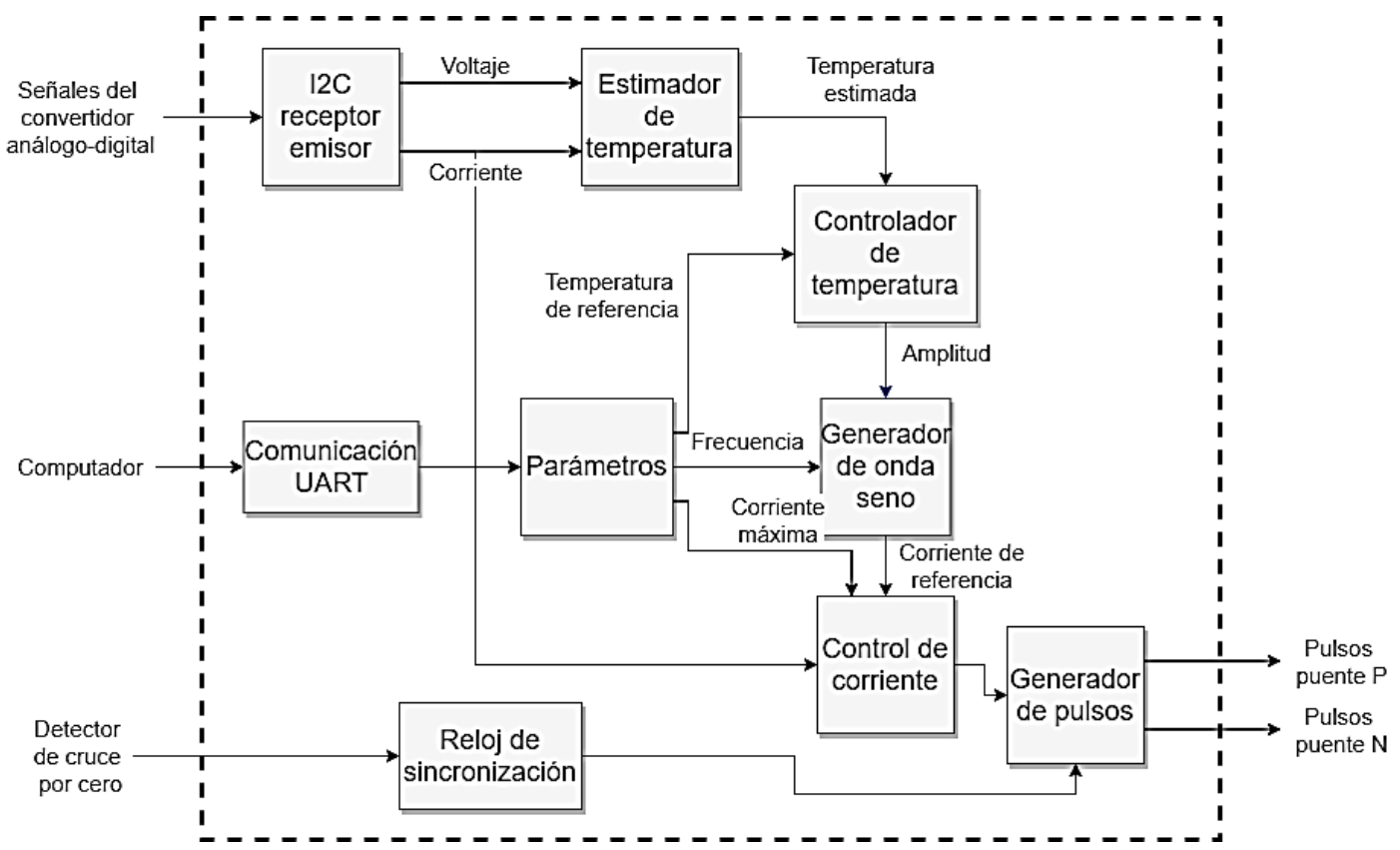

Fig 6: Esquema de los bloques funcionales implementados en la FPGA.

En la FPGA se implementó un circuito de comunicación serial UART que permite enviar los parámetros de trabajo al sistema a través de una interfaz en el computador. En el bloque "Parámetros" se almacenan en registros individuales los valores de referencia ingresados por el usuario. Desde este bloque se impone la temperatura de referencia al "Controlador de temperatura", el cual determina la corriente de salida del sistema LFH y por ende el calentamiento de los devanados del transformador; también le indica al "Controlador de corriente", la corriente máxima que puede entregar el sistema LFH; así mismo, le indica al "Generador de onda seno" la frecuencia de las señales de salida de tensión y corriente del sistema LFH.

El bloque "Control de corriente", a partir de la información de la corriente de referencia y la corriente entregada al transformador, se encarga de proporcionar el ángulo de disparo (a) al "Generador de pulsos". El ángulo de disparo, junto con una referencia del detector de cruce por cero, la cual se procesa previamente a través del "Reloj de sincronización", se introducen al "Generador de pulsos", para que a partir de esta información genere los pulsos de disparo a las diferentes parejas de tiristores del cicloconvertidor. En la Figura 7 se muestra a manera de ejemplo, un conjunto de pulsos de disparos generados desde la FPGA.

\begin{tabular}{|c|c|c|c|c|c|c|c|c|c|c|c|}
\hline \multicolumn{3}{|c|}{ log:Trig @ 2018/09/27 20:01:27 [0: } & \multicolumn{9}{|c|}{ click to insert time bar } \\
\hline Type & Alias & Name & -256 & 0 & 25.6 & 512 & 768 & 1024 & 1280 & 1536 & 1792 \\
\hline$*$ & & $2 x d$ & & & & & & & & & \\
\hline$\frac{7}{6}$ & & ...nce:antirrebote|result & & & & & & & & & \\
\hline 䍃 & & ⿴囗” angle_deg[7..0] & & & & & 2 & & & & \\
\hline$\frac{7}{6}$ & & p1 & & & \|\| & $\|$ & & & & & \\
\hline 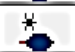 & & $p^{2}$ & & & & $\|$ & & & & & \\
\hline 药 & & p3 & & & & & & $\Perp$ & & & \\
\hline 荫 & & $\mathrm{p4}$ & iు & & & & & $\Perp$ & & & \\
\hline 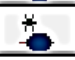 & & p5 & $=$ & iाt & & & & & & & $\Perp$ \\
\hline 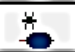 & & $p 6$ & & ill & 㞨 & & & & & & $\Perp$ \\
\hline
\end{tabular}

Fig 7: Pulsos de disparo generados desde la FPGA.

El bloque "I2C receptor emisor" lee la información de la tensión y corriente entregada por el sistema LFH al transformador bajo secado y la comunica a la FPGA mediante el protocolo I2C. Estas señales de tensión y corriente son previamente filtradas y digitalizadas a través de circuitos externos a la FPGA. El bloque "I2C receptor emisor" envía el valor de corriente medido al "Control de corriente" y las señales de tensión y corriente al "Estimador de temperatura", desde donde se determina la temperatura estimada de los devanados del transformador; información que es usada por el bloque "Controlador de temperatura" para determinar el ajuste en la amplitud o valor pico de la señal de corriente, la cual a su vez es tomada como insumo por el bloque "Generador de onda seno", para que este genere una onda de corriente de referencia. 


\section{RESULTADOS Y DISCUSIÓN}

En la Figura 8 se muestra una fotografía en la cual se indican algunos de los componentes, del sistema LFH construido. Además del cicloconvertidor de potencia y de la tarjeta de la FPGA mencionados, se aprecian las tarjetas de encendido de cada uno de los puentes de tiristores, las cuales se sincronizan con el voltaje de AC, que lo alimenta. También se muestran los sensores que miden el voltaje y la corriente de salida del cicloconvertidor y los filtros y conversores análogo-digital (CAD), que adecúan estas señales, así como los elementos de corte y protección (contactores y relés), para la conexión y desconexión de los elementos de potencia, tanto a la carga como a la fuente de alimentación.

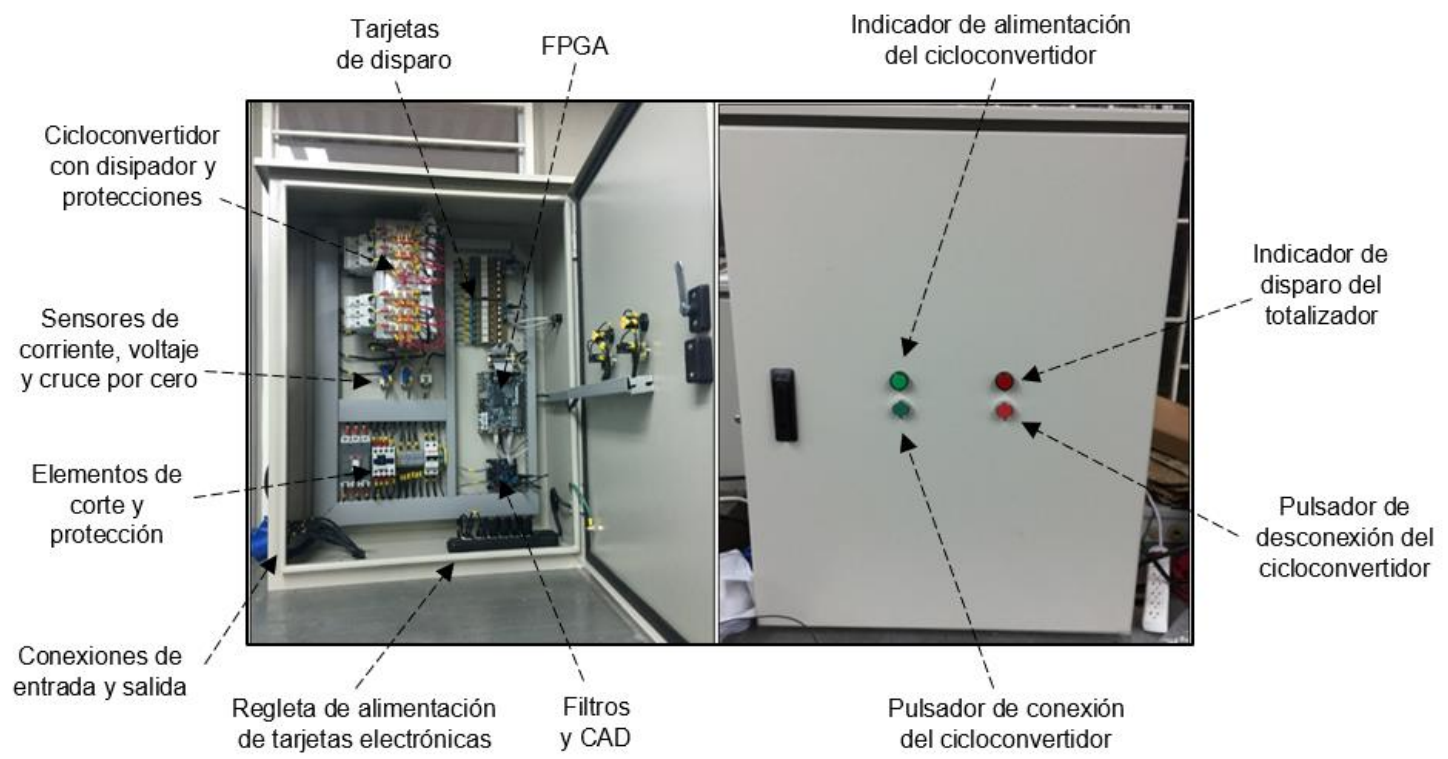

Fig. 8: Foto del equipo LFH construido.

\section{Pruebas y puesta en funcionamiento}

Una vez construido el equipo se le realizaron pruebas de puesta en servicio, inicialmente con una carga constante $R L(R=10 \Omega, L=130 \mathrm{mH})$, con la cual se validó el correcto funcionamiento del equipo, especialmente el control de la magnitud y frecuencia de la corriente. Posteriormente se evaluó el funcionamiento del sistema LFH al usar como carga dos transformadores monofásicos de distribución, el primero de $15 \mathrm{kVA}$ y el segundo de $37,5 \mathrm{kVA}$, ambos con tensiones nominales de $7620 \mathrm{~V} / 240-120 \mathrm{~V}$. Las pruebas con los transformadores permitieron verificar el calentamiento de los devanados de los transformadores como efecto de la corriente de baja frecuencia inyectada, así como el funcionamiento del control indirecto de la temperatura de calentamiento.

En la Figura 9 se muestra la señal de tensión de salida del sistema LFH para una frecuencia de $0,2 \mathrm{~Hz}$, cuando alimenta una carga puramente resistiva, es decir, la corriente tiene similar forma de onda; a la izquierda, se muestra la captura de un par de ciclos, cuando ambos puentes están habilitados, y a la derecha un acercamiento sobre el semiciclo positivo de la onda. En este caso particular, al no existir inductancia, la señal de corriente de salida presenta una alta distorsión.
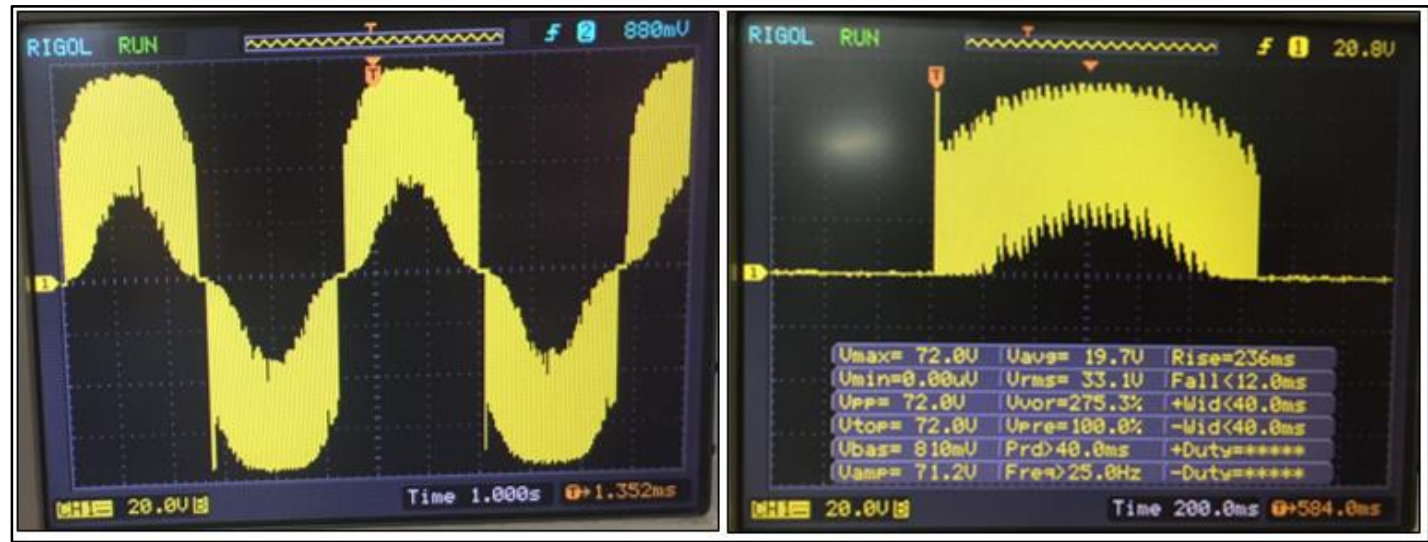

Fig. 9: Oscilogramas de tensión de salida del sistema LFH cuando alimenta una carga puramente resistiva. 
El esquema del montaje de prueba cuando se usa un transformador como carga, se muestra en la Figura 10 y en la Figura 11 se muestra una fotografía del montaje experimental. Ambos transformadores tienen sensores internos de temperatura de fibra óptica (Martínez, Céspedes and García, 2019), con los cuales se realiza la medida directa de la temperatura de los devanados y el aceite, lo que facilitó verificar el efecto de la inyección de corrientes de baja frecuencia en el calentamiento del transformador y el funcionamiento del control indirecto de la temperatura.

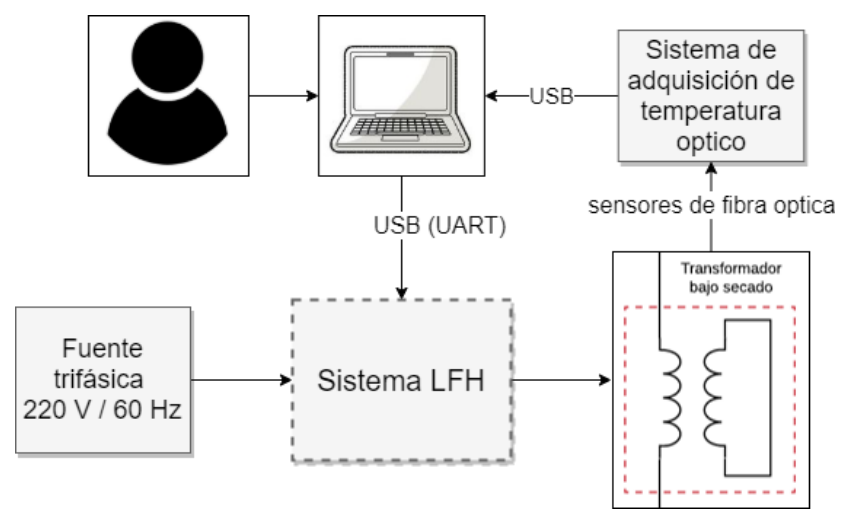

Fig. 10: Esquema del montaje para el calentamiento del transformador.

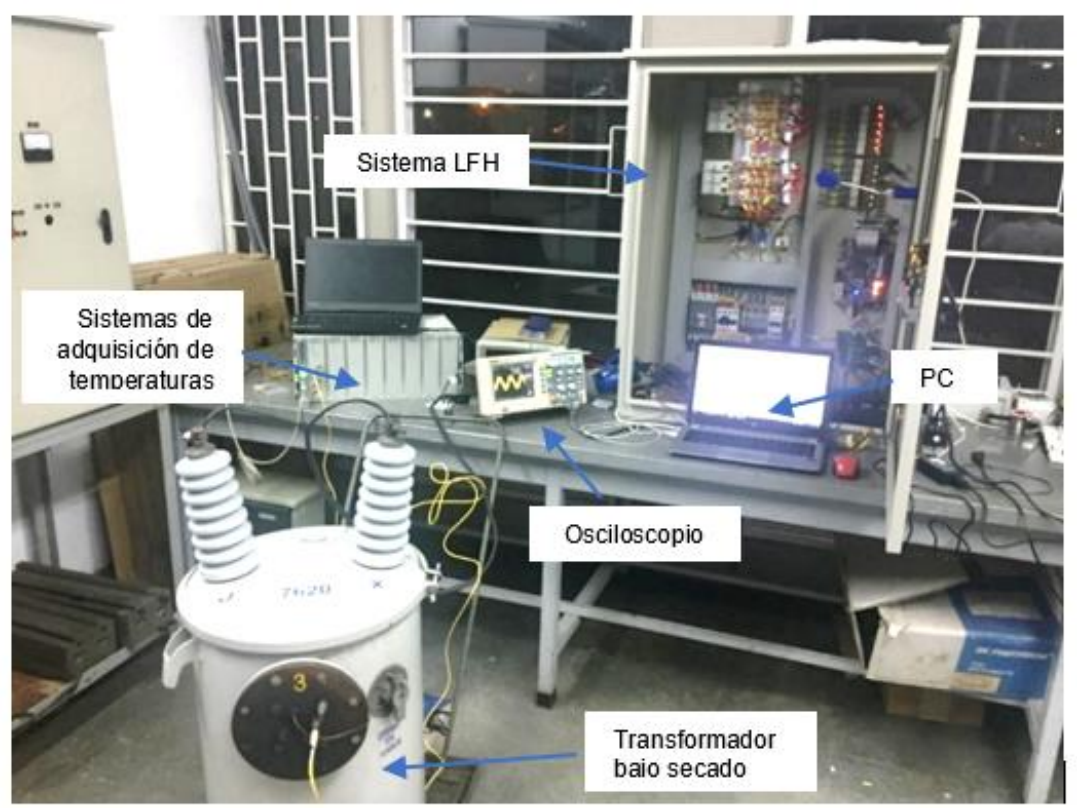

Fig. 11: Esquema del montaje experimental para el calentamiento del transformador de $15 \mathrm{kVA}$.

En la Figura 12 se presentan las formas de onda de tensión que el sistema LFH entrega al transformador de $15 \mathrm{kVA}$; La señal sin filtrar (lado izquierdo) muestra una onda de tensión con envolvente sinusoidal y oscilaciones alrededor de esta envolvente, lo que es normal en el funcionamiento de un cicloconvertidor, cuyo objetivo primordial es generar corrientes con forma sinusoidal. El lado derecho de la imagen muestra la misma onda de tensión, a la cual se le han filtrado las oscilaciones de frecuencias superiores a $50 \mathrm{~Hz}$, es decir, superiores a 10 veces la frecuencia máxima de salida del sistema LFH.

En la prueba se requería aplicar la corriente nominal del transformador para que dicha corriente caliente el devanado primario, y a su vez se induzca en el secundario una corriente que permita el calentamiento de este devanado; sin embargo, en la práctica este transformador mostró gran capacidad de refrigeración, por lo que se decidió aumentar la corriente aplicada aproximadamente un $15 \%$, lo que se logró con un voltaje pico de $73,5 \mathrm{~V}$, en este caso la corriente que circula por el primario es de 2,16 A, por lo que la corriente del devanado secundario fue aproximadamente $60 \mathrm{~A}$.

En la Figura 13 se presenta la señal de corriente sin filtrar, correspondiente al mismo experimento. Se observa que la corriente de salida tiene una forma sinusoidal y con la frecuencia requerida. Las oscilaciones que se observan corresponden al momento en que se produce el cambio del puente de tiristores que entra en operación. La forma de onda antes del filtro es prácticamente igual a la que se obtiene después del filtro, lo que indica que la reactancia del transformador actúa como filtro de la señal de corriente. 


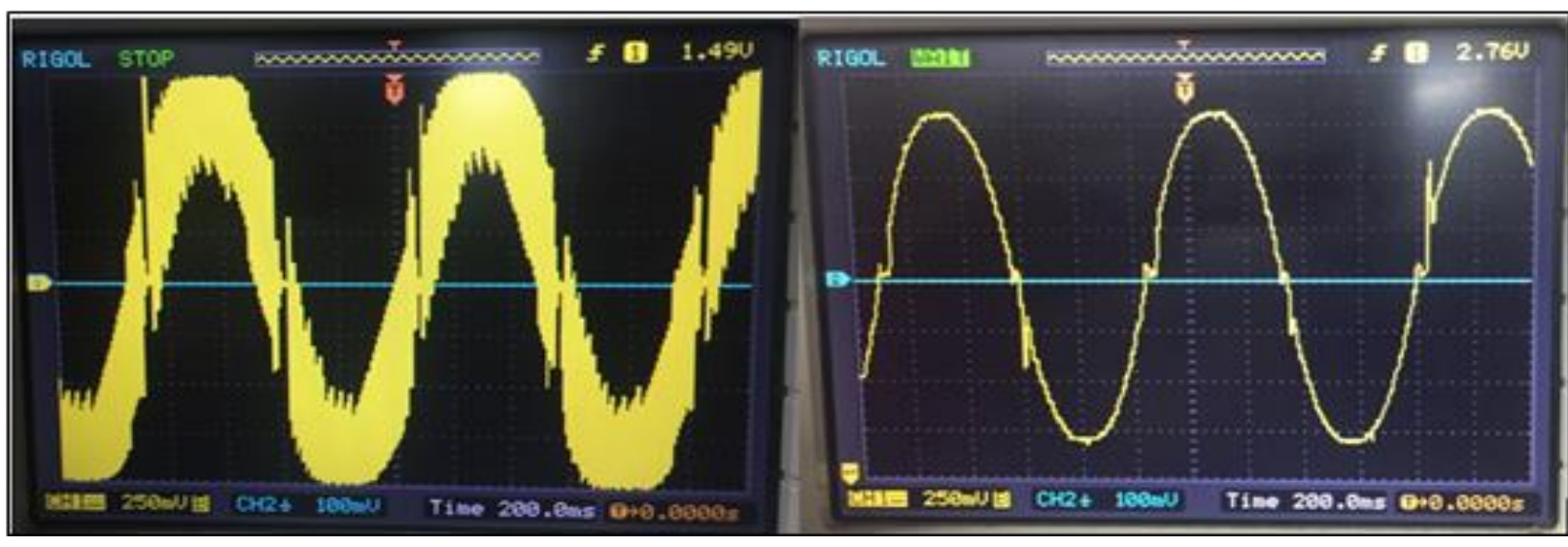

Fig. 12: Forma de onda de la tensión de salida a $1 \mathrm{~Hz}$, lado izquierdo señal sin filtrar y lado derecho señal filtrada.

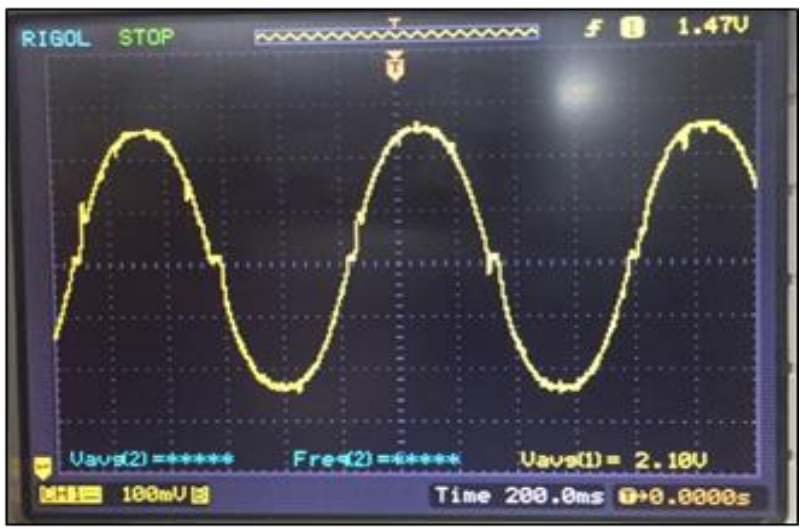

Fig. 13: Oscilograma de la onda de la corriente de salida del sistema $L F H(F=1 \mathrm{~Hz})$.

La validez del sistema LFH para el calentamiento del transformador se comprobó con la medición de temperatura, a la que fueron sometidos los dos transformadores; en primer lugar, se muestra en la Figura 14 la evolución de las temperaturas (devanados y aceite) en el transformador de $15 \mathrm{kVA}$, cuando está en reposo (componentes a temperatura ambiente) y se le inyecta una corriente con una frecuencia de $1 \mathrm{~Hz}$ y magnitud igual a la nominal.

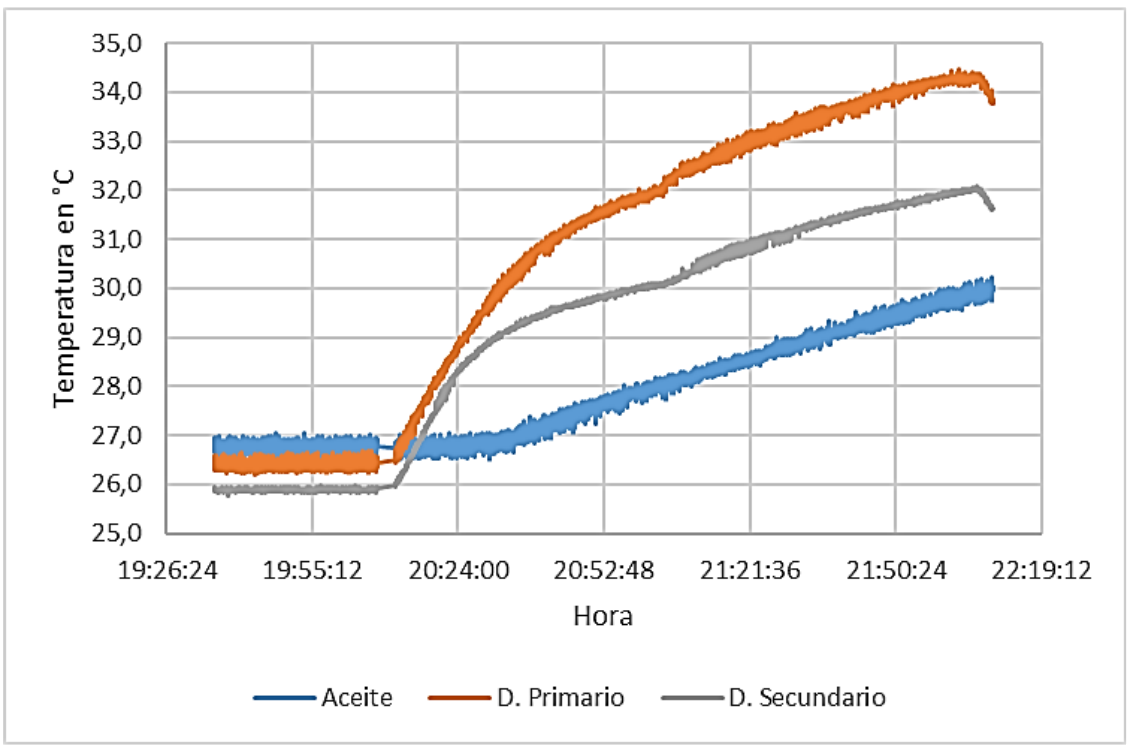

Fig. 14: Evolución de las temperaturas en el transformador de 15 kVA, cuando se le inyecta corriente nominal a $1 \mathrm{~Hz}$.

Se observa que las temperaturas internas de los devanados del transformador crecen, lo que comprueba la validez del sistema para lograr el calentamiento del transformador. Sin embargo, se observó que no era posible obtener en los devanados del transformador una temperatura superior a $45^{\circ} \mathrm{C}$, esto debido a que es práctica común en algunos fabricantes, usar para sus transformadores de menores potencias tanques de 
transformadores de mayor potencia, con lo cual el volumen de aceite refrigerante del transformador es proporcionalmente mayor y por ende tienden a ser transformadores que a carga nominal tienen menor elevación de temperatura en sus devanados y en el aceite.

El transformador de $37,5 \mathrm{kVA}$, el cual tiene un volumen de aceite proporcionalmente menor al primer transformador, se conectó durante 5,5 días, obteniendo los resultados que se muestran en la Figura 15. De allí se observa que el sistema LFH responde muy bien a los cambios programados de temperatura, para lo cual se tuvo en cuenta que la evolución dinámica de la temperatura del transformador es lenta.

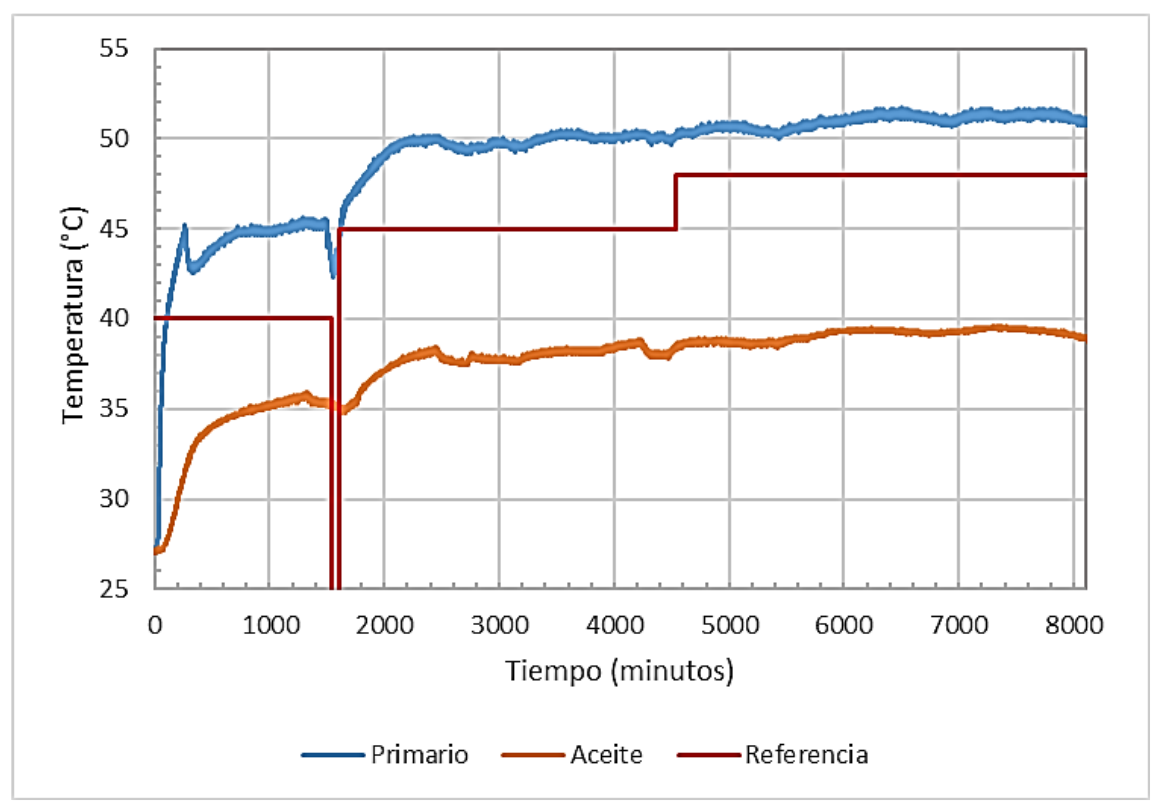

Fig. 15: Gráfico de las temperaturas del transformador de 37,5 kVA registradas al inyectarle corriente con una frecuencia de $1 \mathrm{~Hz}$.

En la Figura 15 se observa además que la temperatura de los devanados del transformador se incrementa con cada incremento en el valor de referencia; sin embargo, existe una diferencia entre el valor de temperatura de referencia y el valor de temperatura alcanzado por el devanado. Esta diferencia entre la temperatura de referencia y la del devanado se explica porque el sensor de temperatura de fibra óptica, que se utiliza para medir la temperatura del devanado primario (devanado más caliente), está ubicada en la posición del punto caliente del transformador (hot-spot), es decir, donde la temperatura del devanado alcanza su máximo valor; mientras que la estimación de la temperatura que realiza el sistema LFH es el promedio de temperatura del devanado.

Al comparar el resultado de la estimación instantánea de temperatura que hace el sistema LFH, y la medida directa de la temperatura, se observa que la primera es varios grados más alta que el valor medido, pero la diferencia entre estos valores se hace más pequeña a medida que se eleva la temperatura, lo cual corresponde con el comportamiento térmico de los transformadores sumergidos en aceite con sistema de refrigeración por circulación natural del aceite y el aire (ONAN), en el cual, al incrementar la carga del transformador, el gradiente de temperatura entre el promedio del devanado y su punto caliente se disminuye. Lo anterior permitió comprobar la validez del funcionamiento del sistema LFH desarrollado, pues se logró controlar el incremento de temperatura en los devanados del transformador mediante la aplicación de corrientes de baja frecuencia.

Finalmente, a partir de la estructura de potencia propuesta, así como las estrategias de control del prototipo del sistema LFH desarrollado y validado a través de este trabajo, se realizó el escalamiento tecnológico a un prototipo industrial trifásico de $250 \mathrm{~kW}$ (500 A y $480 \mathrm{~V}$ de salida), con el cual es posible realizar el calentamiento controlado en sitio, de transformadores de distribución y potencia con capacidades de hasta 30 MVA y $66 \mathrm{kV}$ de tensión nominal, lo cual repercutirá en mejoras de los procesos de secado de transformadores en Colombia.

\section{CONCLUSIONES}

Á partir de la construcción del prototipo electrónico para el calentamiento de la parte activa de transformadores eléctricos monofásicos por el método de LFH, se concluye que: 1) el sistema construido es versátil, ya que permite generar corrientes sinusoidales con frecuencias en el rango de $0,1 \mathrm{~Hz}$ hasta $5 \mathrm{~Hz}$ adecuadas para el 
calentamiento LFH de transformadores bajo secado; 2) se utilizó un cicloconvertidor trifásico, que permitió la entrega al transformador bajo secado, de corrientes sinusoidales con reducida distorsión armónica de hasta $10 \mathrm{~A}$; 3) el desarrollo del sistema de control del equipo se realizó empleando una FPGA; la principal ventaja encontrada al usar este tipo de sistema digital, es la capacidad de construir soluciones de hardware que trabajan en paralelo, lo que permite manejar gran cantidad de señales de activación de los semiconductores, garantizando que las funciones de control, mando y limitación del sistema estén disponibles en todo momento, sin interrupciones; 4) adicionalmente, en el sistema LFH se implementó el control indirecto de temperatura de los devanados del transformador, lo que garantiza que este sistema pueda emplearse como coadyuvante en los procesos de secado de transformadores eléctricos.

\section{AGRADECIMIENTOS}

Los autores agradecen a la Universidad del Valle por su apoyo para la realización de este trabajo a través de la financiación del proyecto interno de investigación Cl 2905 "Sistema electrónico para calentamiento a baja frecuencia (Low Frequency Heating) como coadyuvante en procesos de secado de transformadores eléctricos".

\section{REFERENCIAS}

Cerón, A. F. y otros tres autores, Índice de Salud para Transformadores de Potencia Inmersos en Aceite Mineral con Voltajes entre 69 kV y 230 kV usando Lógica Difusa, Información Tecnológica. 26(2), 107-116 (2015).

Dahlund, M. y otros tres autores, Life Extension of Power Transformers Oil Regeneration, On Site Drying and Onsite Repair, PdMSA Conference (2010).

Figueroa, E., Kalicki, T. y TeNyenhus, E., Low Frequency Heating Field dry-out of a 750 MVA 500 kV autotransformer, Electricity Today, 8-10 (2009).

García, B., García, D. F. y Burgos, J. C., Analysis of the Influence of Low-Frequency Heating on Transformer Drying - Part 2: Experiences with a Real Transformer, doi: 10.1016/j.ijepes.2011.12.023, International Journal of Electrical Power and Energy Systems, 38(1), 90-96 (2012).

García, D. F., García, B. y Burgos, J. C., Modeling Power Transformer Field Drying Processes, doi: 10.1080/07373937.2010.548616, Drying Technology, 29(8), 896-909 (2011).

IEEE Std C57.12.90-2015 (Revision of IEEE Std C57.12.90-2010), IEEE Standard Test Code for Liquid-Immersed Distribution, Power, and Regulating Transformers, doi: 10.1109/IEEESTD.2016.7428800, IEEE, 1-120 (2016).

Koestinger, P., Aronsen, E. y Boss, P., Practical Experience With the Drying of Power Transformers in the Field, Applying the Lfh ${ }^{\star *}$ Technology, Cigré, Report A2-, 8 (2004).

Koestinger, P. y Bruarøy, T., Drying of Power Transformers in the Field, Applying the LFH-Technology in Combination with Oil Reclamation, in CIGRE Paris Session (2006).

Martínez, D. F., Céspedes, A. y García, D. F. Evaluación de Modelos Térmicos usados para Estimar la Temperatura del Punto Caliente en Transformadores de Distribución, https://dx.doi.org/10.4067/S0718-07642019000300295, Información Tecnológica. 30 (3), 295-306 (2019).

Mohan, N., Undeland, T. M. y Robbins, W. P., Electronica de potencia. Convertidores, aplicaciones y diseño, pp 391-393, McGraw Hill, Mexico (2009).

Naimi, M., Farhangi, S. y Ghaemi, R., Thermal Model and Temperature Control of a 30 MVA Distribution Transformer Applied in Low Frequency Drying Process, doi: 10.1109/ccece.2003.1226454, Canadian Conference on Electrical and Computer Engineering, 1, 539-542 (2003).

Skinner, B., Variable Speed Drives - Out of the Box, in South African Sugar Technologists' Association, 488-494 (2013).

Vuolle, R. y Rindlisbacher, G., Abaratar y secar: el procedimiento de secado por baja frecuencia reduce a la mitad los costes de energía, Revista ABB. ABB, (3), 66-69 (2005).

Zhu, X. y otros tres autores, Low-frequency Transformer Heating Source Based on Passivity Control, Electric Power Automation Equipment, (6), 26 (2015). 